\title{
Targeting extracellular pyrophosphates underpins the high selectivity of nisin
}

\author{
BOYAN B. BONEV, ${ }^{*}, \S, 1$ EEFJAN BREUKINK, ${ }^{\dagger}$ E. SWIEZEWSKA, ${ }^{\ddagger}$ BEN DE KRUIJFF ${ }^{\dagger}{ }^{\dagger}$ AND \\ ANTHONY WATTS* \\ *Biomembrane Structure Unit, Department of Biochemistry, University of Oxford, Oxford, UK; \\ ${ }^{\dagger}$ Department Biochemistry of Membranes, Institute of Biomembranes Utrecht University, Utrecht, \\ Holland; ${ }^{+}$Institute of Biochemistry and Biophysics, Polish Academy of Sciences, Warsaw, Poland; and \\ ${ }^{\S}$ School of Biomedical Sciences, University of Nottingham, Nottingham, UK
}

\begin{abstract}
The spread of infectious diseases and the increase in antibiotic resistance represent a lifethreatening global development that calls for new approaches to control microorganisms. Of all potential targets, the essential and unique pathway of bacterial cell wall synthesis, targeted by the first known antibiotic penicillin, remains a perfect candidate for the development of new antibiotics. Here we show that the lantibiotic nisin exercises its antibacterial action by targeting peptidoglycan intermediates' extracellular pyrophosphate, unique to bacterial cell wall precursors. We show that nisin sequesters cell wall precursors found in the outer leaflet of bacterial plasma membranes, Lipid II and undecaprenyl pyrophosphate, into stable complexes. We propose a model of antibacterial action for nisin in which the terminal amino group of Ile1 targets the pyrophosphate groups of the bacterial cell wall precursors, where it docks via a hydrogen bond. The pyrophosphate moiety, a highly conserved chemical group different from the L-Lys-D-Ala-D-Ala docking motif for vancomycin, has no biochemical analogs with comparable properties and is unlikely to be susceptible to bacterial adaptations akin to those responsible for resistance to penicillins and vancomycin.-Bonev, B. B., Breukink, E., Swiezewska, E., de Kruijff, B., Watts, A. Targeting extracellular pyrophosphates underpins the high selectivity of nisin. FASEB J. 18, 1862-1869 (2004)
\end{abstract}

Key Words: lantibiotic $\cdot$ staphylococcal resistance $\cdot$ penicillin binding protein $\cdot$ vancomycin

Resistance to ANTibiotics has become a major problem in the contemporary treatment of infectious diseases. Penicillin resistance in pneumococci, for example, is now common (1), and enterococcal and staphylococcal resistance to vancomycin have been reported $(2,3)$. These antibiotics interfere with the biosynthesis of bacterial cell walls by blocking the active sites of enzymes involved in cell wall formation (e.g., penicillin binding proteins) or by binding directly to cell wall precursor molecules (e.g., Lipid II for vancomycin). Another class of bactericidal peptides, the bacterial lantibiotics, also target peptidoglycan forma- tion. So far, lantibiotics have found use mainly in the control of food-borne pathogens, and selective lantibiotic pressure has not been applied systematically to important human pathogens (e.g., S. aureus or $S$. pneumoniae). This presents them as a new alternative to the conventional cell wall inhibitors, to which resistance has become alarmingly common, and as a model system for the development of new classes of cell wall inhibitors.

The cell walls of bacteria consist of long-chain oligosaccharides cross-linked via polypeptide bridges (peptidoglycan). The peptidoglycan network encapsulates the bacterial plasma membranes, gives it structural stability and, in this way, protects bacteria from the osmotic challenges of their environment. The peptidoglycan is a dynamic system in which degradation by autolysins and incorporation of new peptidoglycan monomers are tightly coupled and controlled.

The final part of bacterial cell wall synthesis, incorporation of glycopeptide monomers into the peptidoglycan (Fig. 1), takes place on the outer leaflet of the bacterial membrane. It is therefore relatively accessible and an excellent target for antibacterial action. The building block of the cell wall, the N-acetylglucosamylN-acetylmuramyl pentapeptide (GlcNAc-MurNAc-5Pep), is synthesized in the cellular interior in two steps. In the first step, MurNAc-5Pep-phosphate from UDPMurNAc-5Pep carrier is attached to undecaprenyl phosphate (11P) located on the inner leaflet of the bacterial membrane (Lipid I). Lipid I is then converted into Lipid II after acquisition of a GlcNAc moiety form, UDP-GlcNAc. Lipid II (Fig. 2) (4) is translocated to the outer membrane leaflet via an unknown mechanism. The disaccharide-pentapeptide is then incorporated into the oligosaccharide chains of the peptidoglycan and cleaved from the pyrophosphate. The remaining carrier phospholipid, undecaprenyl pyrophosphate, $11 \mathrm{PP}$, is recycled to the membrane interior and dephosphorylated to undecaprenyl monophosphate (11P),

\footnotetext{
${ }^{1}$ Correspondence: School of Biomedical Sciences, University of Nottingham, Nottingham, NG7 2UH, UK. E-mail: Boyan.Bonev@nottingham.ac.uk

doi: 10.1096/fj.04-2358com
} 


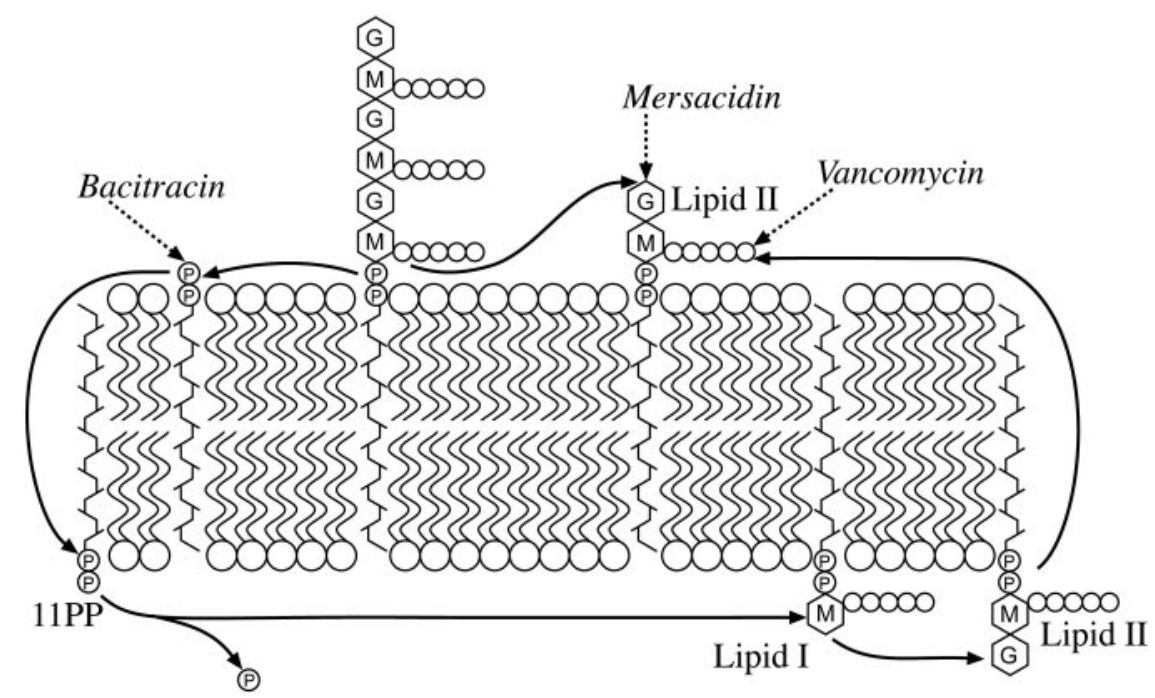

Figure 1. Schematic of peptidoglycan synthesis.

where it re-enters the precursor biosynthesis cycle (5). During these final stages of the cell wall synthesis cycle, two pyrophosphate-containing cell wall precursors, Lipid II and 11PP, reside on the outer leaflet of the bacterial membrane.

A number of antibiotics target the pathway of bacterial cell wall synthesis. Penicillins and cephalosporins inhibit transpeptidation by direct binding to transpeptidases (6), vancomycin hinders cross-peptidation by binding to the key in this process C-terminal L-Lys-DAla-D-Ala residues of the pentapeptide of Lipid II (7), and bacitracin prevents $11 \mathrm{PP}$ form re-entering the biosynthetic cycle (8).

A widely used food preservative (E234), the peptide lantibiotic nisin $(9,10)$ (Fig. 3) produced by Lactococcus lactis, also targets bacterial cell wall synthesis in its bactericidal action $(11,12)$. Nisin is a positively charged peptide that is able to bind to negatively charged plasma membranes (13-15) via nonspecific electrostatic interactions. The antibacterial activity of nisin results from pore formation in the bacterial plasma membrane, which leads to dissipation of the transmembrane potential and vital solute gradients $(16,17)$. The high efficiency of pore formation is the result of a unique mechanism involving the cell wall precursor Lipid II. Lipid II significantly increases the affinity of nisin for the membrane $(11,18)$, stabilizes a transmembrane orientation of nisin (19), and forms and integral part of the nisin pore (20). Due to this high affinity of

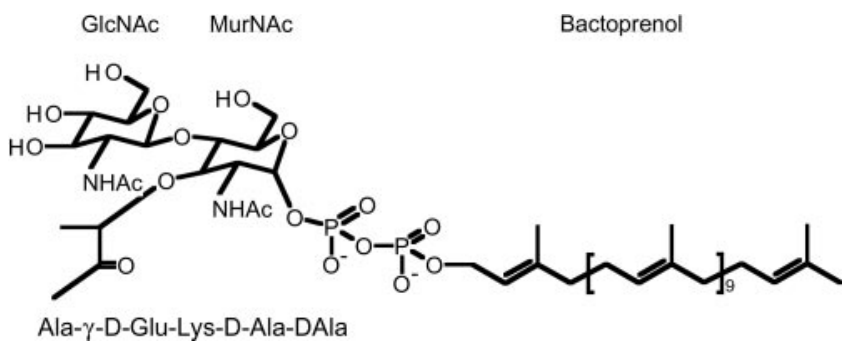

Figure 2. Chemical structure of the cell wall intermediate Lipid II. nisin for Lipid II, nisin variants incapable of permeabilizing the membrane, still exercise bactericidal action by sequestering Lipid II form the cell wall biosynthesis; therefore, nisin has been proposed to possess a dual mode of action (18).

Here we investigate the mechanism of molecular target recognition by nisin during its bactericidal action and, more specifically, the molecular association of the peptide with pyrophosphate-containing cell wall intermediates on the outer leaflet of bacterial membranes. We propose a new method for assaying intermolecular association within lipid bilayers based on the use of solid-state NMR spectroscopy. We used the new assay in combination with a carboxyfluorescein leakage assay to identify 11PP as a target for nisin in addition to Lipid II and an additional mode of action for nisin. The use of NMR and leakage assays made possible the distinction between binary molecular complex formation from lytic pore formation. We suggest that nisin recognizes Lipid II via a two-site docking mechanism. We found that the presence of a pyrophosphate was essential to the affinity of nisin for the cell wall precursors. The binding site within nisin responsible for the interaction of nisin with the pyrophosphate was shown to be the N-terminal Ile1-amine. We suggest that a secondary binding site for nisin exists on Lipid II that is different from the pyrophosphate group.

\section{MATERIALS AND METHODS}

Nisin was isolated and purified as described in ref 21; $\left[{ }^{15} \mathrm{~N}\right]$-nisin was isolated and purified similarly from nisin producing bacteria grown on rich medium with ${ }^{15} \mathrm{~N}$ as the sole nitrogen source. Nisin was permethylated by reductive methylation of the $\varepsilon$-amino group of the lysine residues as well as the amino group of the $\mathrm{N}$ terminus (22), following the same procedure described (13), with the exception that in order to obtain complete methylation, a large (20-fold) molar excess of formaldehyde over nisin was used. The presence of eight methyl groups in the permethylated nisin was confirmed by mass spectrometry. Lipid I and Lipid II were 
Figure 3. Structure of nisin $\mathrm{Z}$.

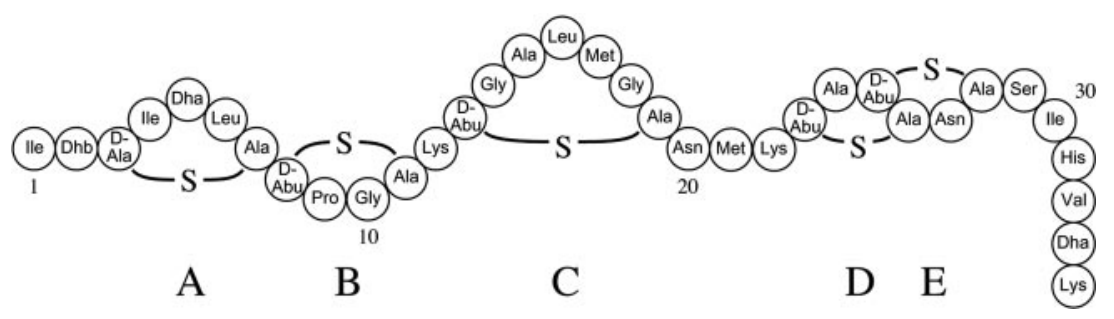

produced following a semisynthetic method (20) from 11-P and 11-PP. The latter two precursors were obtained from phosphorylation of undecaprenol isolated from Laurus nobilis according to ref 23. Synthetic dioleoyl phosphatidylcholine (DOPC) and dioleoyl phosphatidylglycerol (DOPG) were purchased from Avanti Polar Lipids at $>98 \%$ purity and used without further purification.

Samples for NMR experiments were prepared by codissolving DOPC, DOPG (3:1 molar ratio) and 1 molar $\%$ of the corresponding cell wall precursor in a 1:1 chloroform/ methanol mixture. The solvent was removed under vacuum and the dry lipid films were hydrated in nisin-containing buffer (10 mM MES, $100 \mathrm{mM} \mathrm{NaCl,} 1 \mathrm{mM}$ EDTA, pH 6.0). Multilamellar vesicles were obtained by 5 -fold freeze-thawing between liquid nitrogen and a $50^{\circ} \mathrm{C}$ water bath. The samples were pelleted by $1 \mathrm{~h}$ centrifugation at 400,000 $\mathrm{g}$ and the pellet was loaded into $6 \mathrm{~mm}$ magic angle spinning (MAS) NMR zirconia rotors (Chemagnetics/Varian, Palo Alto, CA, USA).

Carboxyfluorescein leakage measurements were performed as described (18) using large unilamellar DOPG/ DOPC (1:3 molar ratio) vesicles containing $1 \mathrm{~mol} \%$ peptidoglycan precursor.

High-resolution cross-polarization (CP) MAS NMR $(24,25)$ experiments were performed on a CMX-Infinity 400 spectrometer equipped with a $6 \mathrm{~mm}$ MAS HX Apex probe (Chemagnetics/Varian). A single $62.5 \mathrm{kHz}$ pulse was used to excite the entire ${ }^{31} \mathrm{P}$ nuclear population and the FID was acquired under $37 \mathrm{kHz}$ continuous wave proton decoupling with a $5 \mathrm{~s}$ interpulse delay. Cross-polarization MAS experiments, during which $92.5 \mathrm{kHz}$ proton excitation was followed by $1 \mathrm{~ms}$ of $65 \mathrm{kHz}$ contact field and $55 \mathrm{kHz}$ TPPM proton decoupling (26) during acquisition and $2 \mathrm{~s}$ interpulse delay, were used to excite only relatively immobile ${ }^{31} \mathrm{P}$ nuclei. Heteronuclear dipolar recoupling experiments, REDOR (27), were performed on a CMX-Infinity 500 spectrometer equipped with a $6 \mathrm{~mm}$ MAS HXY T3 probe (Chemagnetics/ Varian). Proton excitation was achieved using a $111 \mathrm{kHz}$ pulse, followed by $1 \mathrm{~ms}$ of $58 \mathrm{kHz}$ contact field and $52 \mathrm{kHz}$ TPPM decoupling during acquisition. A $62.5 \mathrm{kHz}$ field was used for refocusing ${ }^{15} \mathrm{~N}$ pulse, and the strength of the (24 to 48) ${ }^{31} \mathrm{P}$ dephasing pulses was $83 \mathrm{kHz}$. Between 65536 and 131072 transients were averaged with an interpulse delay of $2.5 \mathrm{~s}$ and a spectral width of $50 \mathrm{kHz}$. Alternating measurements were taken and averaged independently for the dephased and nondephased experiments. Each FID consisted of 1024 data points at $20 \mu$ s dwell time. Phosphorus-31 spectra were referenced externally to $0 \mathrm{ppm}$ for phosphoric acid $(85 \%)$. The ${ }^{15} \mathrm{~N}$ spectra were referenced externally to ${ }^{15} \mathrm{NH}_{3} \mathrm{Cl}$ and chemical shifts are reported relative to TMS.

\section{RESULTS}

High-resolution solid-state ${ }^{31} \mathrm{P}$ MAS NMR spectra from model membranes, composed of DOPC/DOPG at 3/1 molar ratio and containing $1 \%$ of the different precursor molecules (Lipid II, Lipid I, 11PP, and 11P, respec- tively), showed that phosphates in all of the membranereconstituted precursors examined possess a high degree of motional freedom. The molecules of the lipid matrix, DOPC and DOPG, undergo fast rotation along preferred molecular axes, which results in a partial averaging of the chemical shift anisotropy of their phosphates from a 200 ppm value, observed from immobile phosphates to $\sim 40 \mathrm{ppm}$. Under $5 \mathrm{kHz}$ MAS conditions, this is seen as the presence of single, low intensity rotational sidebands separated from the principal high-resolution lines by the magnitude of the rotation speed. The absence of rotational sidebands from any of the membrane-incorporated precursors even at very low rotational speeds $(<800 \mathrm{~Hz}$; not shown) reveals the presence of additional motional freedom in the pyrophosphate/phosphate region (Fig. $4 A)$. Such motional freedom facilitates the complete averaging of the residual CSA and only a central high-resolution resonance is observed from each precursor phosphate. The dipolar couplings between the phosphates and the protons located in their vicinity are averaged, and the pyrophosphates are not observed after cross-polarization (Fig. 4B).

Individual phosphates from the pyrophosphate groups in Lipid II and Lipid I were well resolved at -8 and $-10 \mathrm{ppm}$. The two phosphates in 11PP produce overlapping lines at $-10 \mathrm{ppm}$. The longitudinal relaxation times of the lipid matrix and the precursor were within $1 / 5$ of the oversampling delay time and a single pulse excitation, followed by proton decoupling, was used for acquisition. The isotropic chemical shift values of the principal resonances from each phospholipid are summarized in Table $\mathbf{1}$.

Nisin was added in consecutive experiments to DOPC/DOPG bilayers containing $1 \%$ of Lipid II, Lipid I, $11 \mathrm{PP}$, or $11 \mathrm{P}$ at $2 / 1$ molar excess to each precursor. The ${ }^{31} \mathrm{P}$ MAS NMR spectra, acquired from Lipid IIcontaining bilayers after a single pulse excitation, showed only matrix DOPC and DOPG resonances (Fig. $4 C$ ). The resonance lines arising from the Lipid II phosphates were saturated under the $5 \mathrm{~s}$ pulse repetition due to an increase in their ${ }^{31} \mathrm{P}$ longitudinal relaxation time.

The Lipid II phosphates were well resolved in a short mixing time (1 ms) cross-polarization experiment (Fig. $4 D)$, where excitation of the matrix DOPC and DOPG phosphates was less efficient (optimal mixing at 10-15 $\mathrm{ms})$. Four orders of sidebands, bracketing the pyrophosphates' center bands, were resolved under $5 \mathrm{kHz}$ MAS. The overall width of the phosphate spectrum was 

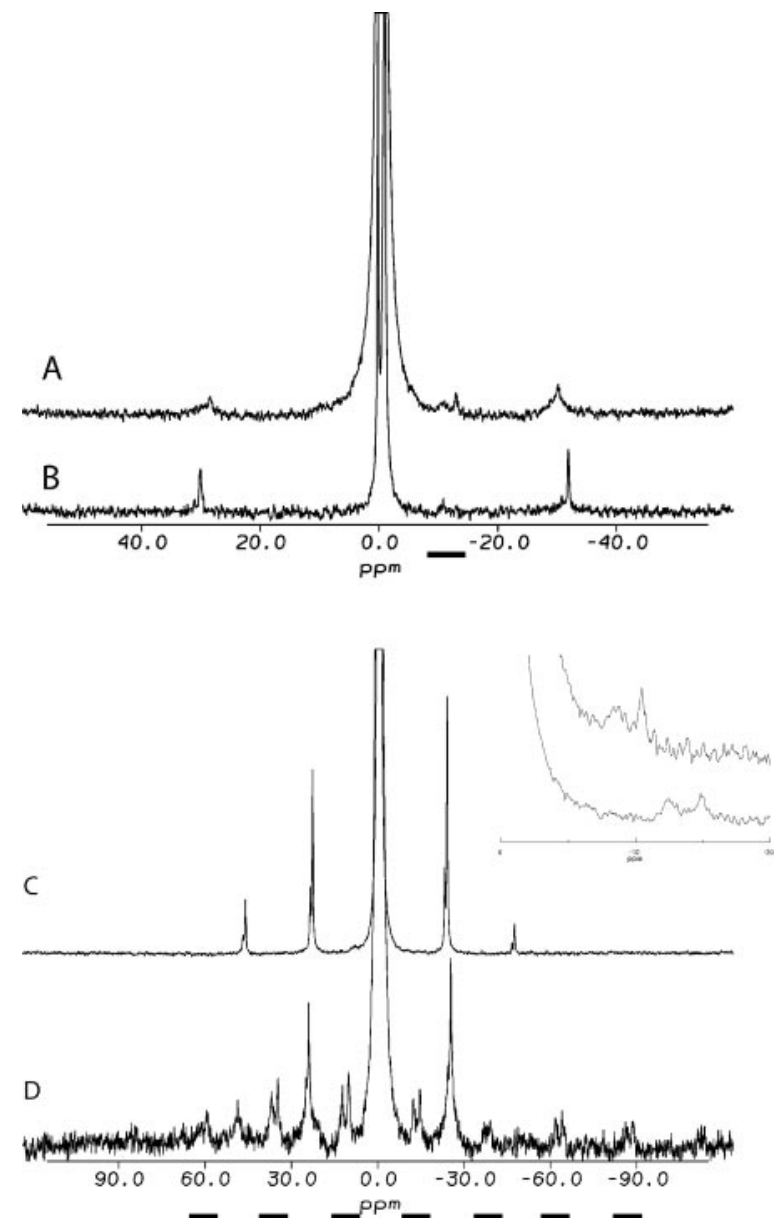

Figure 4. High-resolution ${ }^{31} \mathrm{P}$ MAS NMR spectra from fluid bilayer membranes of 3:1 DOPC/DOPG containing 1\% Lipid II after single pulse excitation $(A)$ and CP $(B)$. The spectra from the same mixture after 2:1 molar addition of nisin to Lipid II are shown after single pulse excitation $(C)$ and CP $(D) . D)$ Rotational sidebands in the pyrophosphate spectrum are underlined; inset shows the centreband of the pyrophosphate in Lipid II (spectra A and D). All spectra were acquired at $30^{\circ} \mathrm{C}$ and the spinning speed was 4.7 and $5 \mathrm{kHz}$ for the single pulse and the CP experiments, respectively.

$\sim 200$ ppm, which corresponds to the typical CSA of immobile phosphates. This dramatic increase in the observed CSA, as well as the increase in ${ }^{31} \mathrm{P} \mathrm{T}_{1}$, reflects the presence of severe motional restrictions imposed on the pyrophosphates by binding of Lipid II to nisin. Such loss of molecular mobility allows for a high efficiency of ${ }^{31} \mathrm{P}$ and ${ }^{1} \mathrm{H}$ cross-polarization during the short mixing time, which contributes to the relatively high intensity of the pyrophosphates observed in comparison to the DOPC/DOPG lipid matrix. The bilayer matrix lipids DOPC and DOPG underwent only subtle changes in response to the addition of nisin (cf. ref 15), which shows the strong affinity of nisin for Lipid II. This selective immobilization of Lipid II by nisin is a clear evidence of the formation of nisin/Lipid II molecular complexes of monomeric or oligomeric nature. Besides the dramatic increase in effective CSA, the addition of nisin caused a change in the chemical shift of the two phosphates of 2 ppm downfield. This suggests the presence of a positive charge in the immediate vicinity of the pyrophosphate moiety (28). Similar results were obtained when Lipid II was replaced by Lipid I, indicating that the GlcNAc moiety of Lipid II does not play a role in the interaction of nisin with Lipid II.

Under conditions used to observe the immobilization of Lipids I and II, we tested for the presence of pores in the model membranes using carboxyfluorescein leakage experiments (Table 2). In both cases, addition of nisin to $1 \mathrm{~mol} \%$ Lipid I- or II-containing vesicles (DOPG/DOPC 1:3 molar ratio) resulted in the rapid efflux of carboxyfluorescein $(\mathrm{CF})$ and a sharp increase in fluorescence intensity. No difference could be observed between the two systems (not shown). Therefore, nisin/Lipid I as well as nisin/Lipid II complexes observed by high-resolution ${ }^{31} \mathrm{P}$ CP MAS NMR permeabilize the lipid membranes via the formation of sufficiently large nonselective oligomeric pores. The observed pore formation by nisin in the presence of either lipid, and the indistinguishable spectroscopic feature of the two precursors suggest that the GlcNAc moiety of Lipid II is not important for pore formation. To test the importance of the MurNAc-pentapeptide moiety for the pore-forming activity of nisin, CF leakage experiments were performed with 1\% 11-PP containing vesicles (DOPG/DOPG 1:3 molar ratio). No leakage of $\mathrm{CF}$ could be detected even in excess of nisin (not shown). Thus, the MurNAc-pentapeptide moiety is absolutely required for the pore-forming activity of nisin.

The lack of pore formation by nisin in 11PP-containing membranes pointed to this compound as a negative control in the complex formation assays, shown for Lipid I and II. Single pulse excitation ${ }^{31} \mathrm{P}$ MAS NMR spectra from $1 \% 11 \mathrm{PP}$ in $\mathrm{DOPC} / \mathrm{DOPG}$ bilayers showed a single resonance at -10 ppm from both phosphates in 11PP (Fig. 5a). This differs from the ${ }^{31} \mathrm{P}$ solution spectrum of $11 \mathrm{PP}$ at $\mathrm{pH} 8$, where the terminal phosphate is observed at -6.7 and the alkylated phosphate at $-9.7 \mathrm{ppm}$, possibly as a result of the substantial difference between the phosphate hydration in solution and the lipid bilayer. Nisin was added at a 2:1

TABLE 1. Isotropic chemical shift in parts per million [ppm] of cell wall precursor phosphates and the membrane matrix ${ }^{a}$

\begin{tabular}{lcccrr}
\hline \hline $\mathrm{CS}_{\text {iso }}[\mathrm{ppm}]$ & LII & LI & $11 \mathrm{PP}$ & $11 \mathrm{P}$ & PC \\
\hline $\begin{array}{l}\text { Membrane } \\
+ \text { Nisin Z }\end{array}$ & $-8,-10$ & $-8,-10$ & $-10,-10$ & 0 & PG \\
\hline
\end{tabular}

${ }^{a}$ Only one precursor was reconstituted into 3/1 molar ratio DOPC/DOPG membranes at a time. 
TABLE 2. Effect of nisin on molecular mobility and permeability of lipid membranes for $\mathrm{CF}$

\begin{tabular}{lcccccc}
\hline \hline Nisin Z + & LII & LI & $11 P P$ & $11 P$ & PC & PG \\
\hline Membrane poration & + & + & - & - & - & - \\
Complex formation & + & + & + & - & - & - \\
\hline
\end{tabular}

molar excess to DOPC/DOPG bilayers, containing $1 \%$ 11PP. The ${ }^{31} \mathrm{P}$ CP MAS spectrum from the pyrophosphate showed a single broad component at $\sim-10 \mathrm{ppm}$, with FWHH of almost $5 \mathrm{ppm}$ and three orders of spinning sidebands (Fig. $5 b$ ). The presence of a high number of spinning sidebands clearly indicates, contrary to our expectation, the formation of stable nisin/ 11PP complexes. The large line width must reflect the lack of a unique binding location on nisin for the pyrophosphate group or of a unique orientation of a putative hydrogen bond with nisin.

The above experiments suggest a specific interaction of nisin with the pyrophosphate. We tested this specificity by examining the interaction of nisin with 11Pcontaining membranes. The bilayer-reconstituted $11 \mathrm{P}$ produced under a single pulse excitation MAS NMR a single resonance, overlapped by the matrix phospholipids, at a chemical shift characteristic of free inorganic phosphate (Fig. $5 c$ ). No changes in this behavior were observed in the presence of nisin either under a single pulse excitation or after CP (Fig. $5 d$ ). Therefore, removal of one of the phosphates completely abolishes the ability of nisin to engage 11-P into membranebound complexes. The lack of nisin induced CF leakage from 11-P doped vesicles confirmed this observation (not shown).

The large downfield shift of the Lipid II phosphates after the addition of nisin is caused by the N-terminal amino group of nisin. We confirmed this after observing by REDOR solid-state MAS NMR a direct internuclear ${ }^{15} \mathrm{~N}-{ }^{31} \mathrm{P}$ dipolar coupling between the $\mathrm{N}$ terminus of uniformly ${ }^{15} \mathrm{~N}$-labeled nisin and the pyrophosphate of Lipid II in the complex (Fig. 6). Nitrogen-15 MAS NMR spectra from uniformly labeled nisin in its membrane-associated complexes with Lipid II were acquired in the presence and in the absence of ${ }^{31} \mathrm{P}$-dephasing pulses. Reduction in the spectral intensity was observed after application of ${ }^{31} \mathrm{P}$-dephasing pulses only for those resonances, which arose from nitrogens directly coupled to phosphates and located within a range of up to $\sim 6 \AA$ from ${ }^{31} \mathrm{P}$ nuclei. The backbone nitrogens produced a largely overlapping set of resonances at $\sim 120$ ppm and could not be assigned in this experiment. The $\varepsilon$-nitrogens from lysines 12, 22 and 34 appear at $34 \mathrm{ppm}$ and the N-terminal amino group of Ile1 nisin is clearly resolved at $40 \mathrm{ppm}$ with $\sim 1 / 3$ of the intensity observed from the overlapping Lys-12, 22 and 34 (Fig. 6). After a train of $24{ }^{31} \mathrm{P}$-dephasing pulses, the intensity of the terminal amino group and not that of the lysines appeared substantially reduced, which indicates that the terminal amino group of Ile1 is directly involved in the docking of nisin onto the pyrophosphates of Lipid
II. In addition, dephasing was observed in residues within the backbone region, which could not be assigned in this experiment.

Direct involvement of the terminal amino group of nisin in the complex with Lipid II and the required presence of an accessible pyrophosphate in the target molecule suggest the possibility of formation of a hydrogen bond between PP on Lipid II and $\mathrm{NH}_{2}$-Ile 1 of nisin. We examined this by doubly methylating the four amino groups of nisin. While the importance of the lysines in nisin has been attributed mainly to their contribution to the overall molecular charge in binding to lipid bilayers, methylation of the $\mathrm{N}$ terminus would prevent its involvement in the formation of hydrogen bonds with Lipid II. Indeed, ${ }^{31}$ P MAS NMR showed that Met-nisin does not have the ability to form complexes with 11PP. Its activity against Gram-positive organisms is severely reduced and does not permeabilize vesicles containing any of the precursors investigated here (not
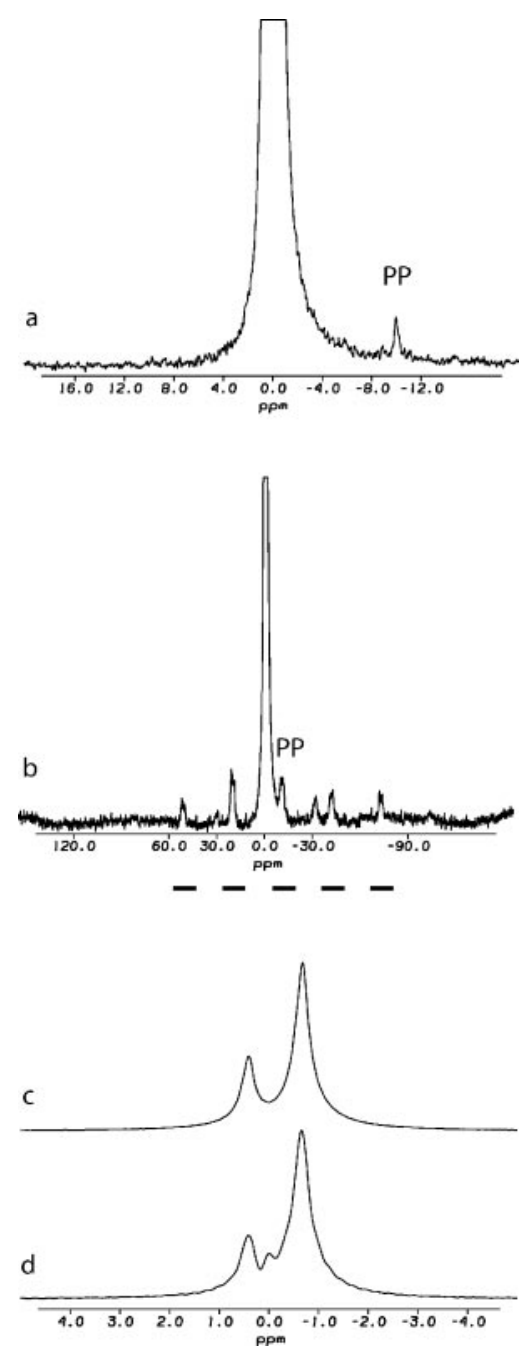

Figure 5. High-resolution ${ }^{31} \mathrm{P}$ MAS NMR spectra from fluid bilayer membranes of 3:1 DOPC/DOPG with 1\% 11PP. One pulse excitation $(a)$; 11PP plus $1: 1$ nisin $\mathrm{Z}, \mathrm{CP}(b) ; 11 \mathrm{P}$, one pulse $(c) ; 11 \mathrm{P}$ plus nisin $\mathrm{Z}, \mathrm{CP}(d)$. All spectra were acquired at $30^{\circ} \mathrm{C}$ and $5 \mathrm{kHz}$ spinning speed. The rotational sidebands in the pyrophosphate spectrum are underlined in panel $b$. 
A

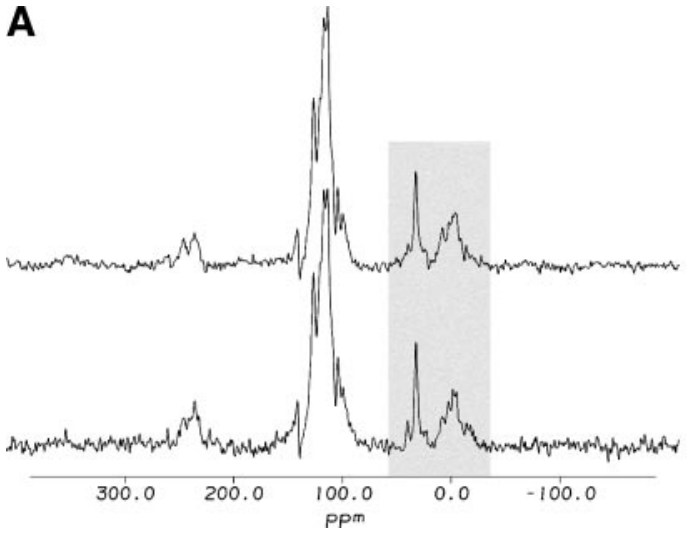

shown). Our ${ }^{31} \mathrm{P}$ MAS NMR experiments showed that Met-nisin does engage Lipid II in stable complexes. The CP MAS spectra (Fig. 7) show the presence of a single broad resonance at $\sim-14 \mathrm{ppm}$ with a line width of over $5 \mathrm{ppm}$, bracketed by sidebands revealing spectral width of $\sim 200 \mathrm{ppm}$. From this we conclude that nisin engages Lipid II in a second binding site.

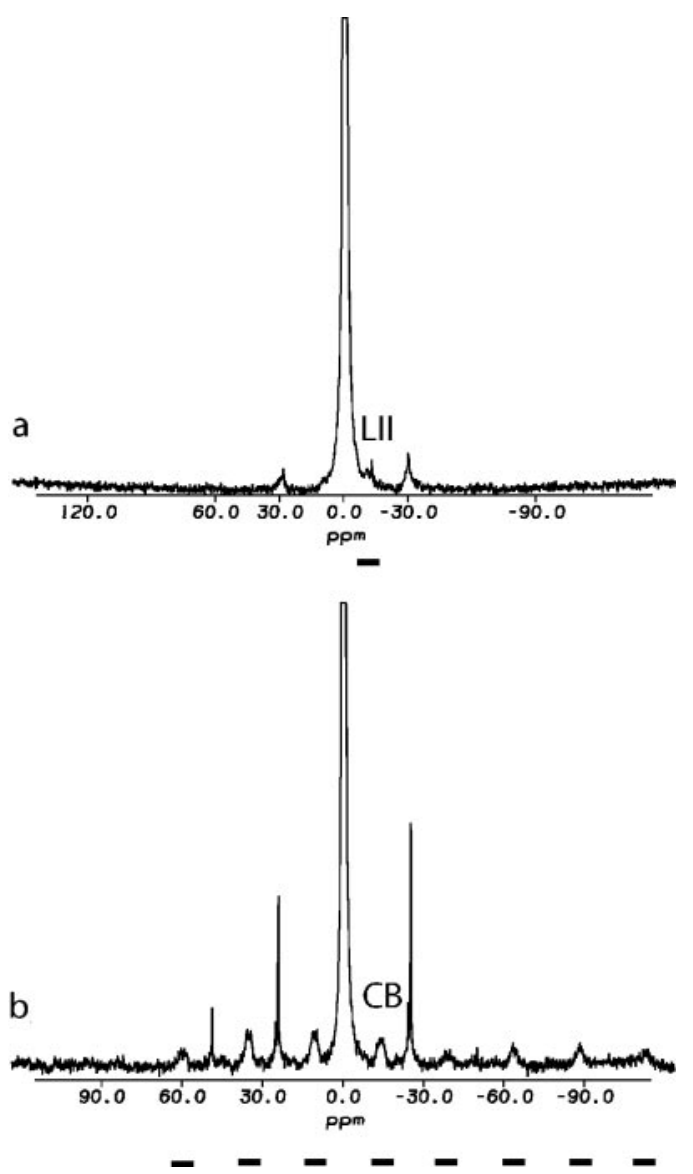

Figure 7. High-resolution ${ }^{31} \mathrm{P}$ MAS NMR spectra from fluid bilayer membranes of 3:1 DOPC/DOPG with 1\% Lipid II after one pulse excitation $(a)$ and in the presence of Metnisin $\mathrm{Z}$ after $\mathrm{CP}(b), 30^{\circ} \mathrm{C}$ and $5 \mathrm{kHz}$ spinning speed. The rotational sidebands in the pyrophosphate spectrum are underlined in panel $b$.

\section{DISCUSSION}

The activity of the bacterial antibiotic nisin is a few orders of magnitude superior to that of similar size membrane-perturbing bactericidal peptides from defense systems of higher organisms. In contrast to the latter, the attack of nisin on bacterial membranes involves specific docking onto Lipid II, an intermediate in the cell wall synthesis pathway, unique to bacteria. Recently we identified the residues in the nisin molecule that are important for its interaction with Lipid II (29), but information on important sites on Lipid II has been lacking. In this report we have identified an important docking motif for nisin on Lipid II.

Our ${ }^{31} \mathrm{P}$ MAS NMR results show in a direct way the formation of a complex between Lipid II and nisin. This is seen as a loss in mobility in the region of the phosphates of Lipid II after addition of nisin to lipid membranes containing the precursor and points to the formation of either monomeric or oligomeric nisin/ Lipid II complexes. Since the formation of nisin/Lipid II complexes was shown in our carboxyfluorescein leakage assays to permeabilize lipid membranes, the existence of oligomeric proteolipid pores emerges as the likely mode of nisin action in the presence of the precursor, in agreement with recent results (20). In contrast, complexes between nisin and 11PP observed in our ${ }^{31} \mathrm{P}$ MAS NMR experiments did not appear to induce membranes leakage, which indicates the existence of monomeric membrane-incorporated nisin/ 11PP complexes. In our ${ }^{31} \mathrm{P}$ MAS NMR and leakage assays, we did not observe differences in the behavior of Lipid II and Lipid I in the presence of nisin, which means that the terminal GlcNAc sugar of Lipid II is not likely to be involved in the interaction with nisin.

The change of the chemical shifts of the pyrophosphate upon binding to nisin and the heteronuclear dipolar recoupling MAS NMR experiments have highlighted the pivotal role of the terminal amino group of nisin in pyrophosphate recognition of Lipid II and in pore formation. Other experiments have emphasized the importance of the amino-terminal region of nisin in that extension of the $\mathrm{N}$ terminus by addition of four residues (ITPQ) has abolished the bactericidal activity of nisin (30). 
The strong reduction of nisin's antimicrobial activity, which follows N-terminal methylation, points to a mechanism of Lipid II recognition that involves the formation of a hydrogen bond between $\mathrm{NH}_{2}$-nisin and PP-Lipid II. This is confirmed by the dipolar dephasing MAS NMR estimate of $6 \AA$ for the ${ }^{15} \mathrm{~N}-{ }^{31} \mathrm{P}$ internuclear distance between ${ }^{15} \mathrm{HN}_{4} \mathrm{Cl}_{3}$ and $\mathrm{P}_{2} \mathrm{O}_{7}$.

Two independent binding sites for nisin exist on Lipid II. One is identified here as the pyrophosphate group; the other must reside on the MurNAc-pentapeptide moiety. The equivalence on Lipid I and Lipid II as nisin targets from an NMR standpoint and as targets for membrane lysis in vitro has ruled out GlcNAc as an association site. Earlier experiments suggest lack of involvement of the terminal D-Ala in nisin binding as well (11). The existence of the second binding site was deduced after we observed a strong association of methylated nisin with Lipid II but not with 11PP. This second site of association alone is not sufficient for pore formation; both sites of contact must be engaged by nisin for the formation of lytic transmembrane pores to occur.

The engagement of pyrophosphate-containing cell wall precursors into stable intermolecular complexes by nisin that takes place on the outer membrane leaflet suggests an additional molecular mechanism of action of nisin whereby the precursor is withdrawn from the biosynthetic pathway by the antibiotic. The existence of such activity has been inferred from mutational analysis, which showed that nisin variants can lose their capability to form pores while retaining bactericidal activity by inhibiting bacterial cell wall synthesis (18).

A third mode of activity is proposed here whereby nisin interferes with peptidoglycan formation by withdrawing 11PP from the biosynthetic pathway into membrane-associated complexes (Fig. 8). The formation of hydrogen bonds makes the association of nisin with Lipid II far stronger than that responsible for the action of the highly toxic antibacterial agent bacitracin (8), which interacts with $11 \mathrm{PP}$ only via electrostatic effects. Indeed, we observed that bacitracin did not restrict
11PP pyrophosphate mobility under similar conditions used for nisin but with added magnesium ions (not shown).

\section{CONCLUSIONS}

We have described the molecular mechanism underpinning the recognition of bacterial membranes by nisin that precedes lytic pore formation. Using solidstate NMR, we have demonstrated that nisin forms stable complexes with Lipid II and 11PP, both of which are present in the outer leaflet of bacterial plasma membranes. The presence of pyrophosphate in the target molecules was found to be essential to target engagement, which suggests the pivotal role this moiety plays in the high activity and low cytotoxicity of nisin. Solid-state NMR revealed a key role of the $\mathrm{N}$ terminus of nisin in target recognition. We have identified a secondary binding site for nisin on Lipid II that is different from the pyrophosphate and not present in 11 PP. We propose a new method for investigation of membrane-associated molecular complexes based on solid-state NMR detection of changes in molecular mobility during complex formation.

A new mode of attack by nisin on the bacterial cell wall biosynthesis is outlined. In the proposed mechanism, nisin engages cell wall intermediates Lipid II and 11PP into stable detergent-insoluble complexes, which are withdrawn from the cell wall biosynthesis. Pore formation and cell wall inhibition involve at molecular level N-terminal binding of nisin to the pyrophosphate moiety of cell wall precursor molecules, located on the outer leaflet of bacterial membranes. The importance of the present findings lies in the demonstration of an attack on extracellular pyrophosphates, which are unique to the bacterial kingdom and whose chemical functions cannot be substituted by simple mutations. Therefore, resistance via target mutation to antibiotics,
Figure 8. Schematic representation of the multiple mode of antibacterial action of nisin. M, MurNAc; G, GlcNAc; P, phosphate. Proposed cell wall inhibition points are indicated with crosses.

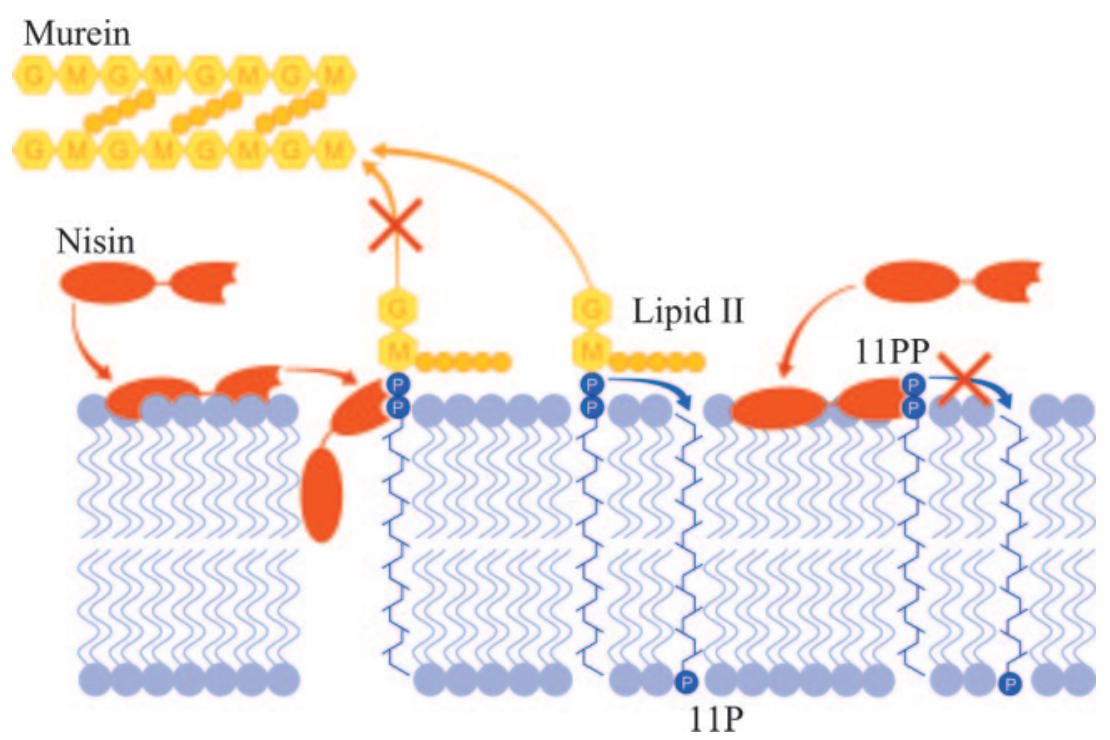


which use pyrophosphates for target recognition, is unlikely to occur.

Funding for instrumental and project support is acknowledged in grants to A.W. from the Higher Education Funding Council for England, the Medical Research Council, the Biotechnology and Biological Sciences Research Council, and Magnex Scientific and Varian Inc. The BBSRC are acknowledged for project funding to B.B. We thank Alex Morgan for preparing Figs. 1, 3, and 8.

\section{REFERENCES}

1. Garau, J. (2002) Treatment of drug-resistant pneumococcal pneumonia. Lancet Infect. Dis. 2, 404-415

2. Wright, G. D. (2003) Mechanisms of resistance to antibiotics. Curr. Opin. Chem. Biol. 7, 563-569

3. Gutmann, M. M. L. (1997) Methicillin-ressitant Staphylococcs aureus and vancomycin-resistant enterococci: therapeutic realities and possibilities. Lancet 349, 1901-1906

4. Van Heijenoort, J. (2001) Recent advances in the formation of the bacterial peptidoglycan monomer unit. Nat. Prod. Rep. 18, 503-519

5. Holtje, J.-V. (1998) Growth of the stress-bearing and shapemaintaining murein sacculus of Escherichia coli. Microbiol. Mol. Biol. Rev. 62, 181-203

6. Tipper, D. J., and Strominger, J. L. (1968) Biosynthesis of the peptidoglycan of bacterial cell walls: inhibition of cross-linking by penicillins and cephalosporins. J. Biol. Chem. 243, 3169-3179

7. Barna, J. C. J., and Williams, D. H. (1984) The structure and mode of action of glycopeptide antibiotics of the vancomycin group. Annu. Rev. Microbiol. 38, 339-357

8. Stone, K. J., and Strominger, J. L. (1971) Mechanism of action of bacitracin: complexation with metal ion and $\mathrm{C}_{55}$-isoprenyl pyrophosphate. Proc. Natl. Acad. Sci. USA 68, 3223-3227

9. Delves-Broughton, J., Blackburn, P., Evans, R. J., and Hugenholtz, J. (1996) Applications of the bacteriocin nisin. Antonie Van Leeuwenhoek 69, 193-202

10. Rogers, L. A., and Whittier, E. O. (1928) Limiting factors in lactic fermentation. J. Bacteriol. 16, 211-214

11. Breukink, E., Wiedemann, I., van Kraaij, C., Kuipers, O. P., Sahl, H.-G., and de Kruijff, B. (1999) Use of the cell wall precursor Lipid II by a pore-forming peptide antibiotic. Science 286, 2361-2364

12. Brotz, H., Josten, M., Wiedemann, I., Schneider, U., Gotz, F., Bierbaum, G., and Sahl, H.-G. (1998) Role of lipid-bound peptidoglycan precursors in the formation of pores by nisin, epidermin and other lantibiotics. Mol. Microbiol. 30, 317-327

13. Breukink, E., van Kraaij, C., Demel, R. A., Siezen, R. J., Kuipers, O. P., and de Kruijff, B. (1997) The C-terminal region of nisin is responsible for the initial interaction of nisin with the target membrane. Biochemistry 36, 6968-6976

14. Breukink, E., Ganz, P., de Kruijff, B., and Seelig, J. (2000) Binding of nisin $\mathrm{Z}$ to bilayer vesicles as determined with isothermal titration calorimetry. Biochemistry 39, 10247-10254
15. Bonev, B. B., Chan, W. C., Bycroft, B. W., Roberts, G. C. K., and Watts, A. (2000) Interaction of the lantibiotic nisin with mixed lipid bilayers: a ${ }^{31} \mathrm{P}$ and ${ }^{2} \mathrm{H}$ study. Biochemistry 39, 11425-11433

16. Sahl, H.-G. (1991) In Nisin and Novel Lantibiotics (Jung, G., and Sahl, H.-G., eds) pp. 347-358, ESCOM Science Publishers, Leiden

17. Chan, W. C., Leyland, M., Clark, J., Dodd, H. M., Lian, L. Y., Gasson, M. J., Bycroft, B. W., and Roberts, G. C. K. (1996) Structure-activity relationships in the peptide antibiotic nisin: antibacterial activity of fragments of nisin. FEBS Lett. 390, $129-132$

18. Wiedemann, I., Breukink, E., van Kraaij, C., Kuipers, O. P., Bierbaum, G., de Kruijff, B., and Sahl, H.-G. (2001) Specific binding of nisin to the peptidoglycan precursor Lipid II combines pore formation and inhibition of cell wall biosynthesis for potent antibiotic activity. J. Biol. Chem. 276, 1772-1779

19. van Heusden, H. E., de Kruijff, B., and Breukink, E. (2002) Lipid II induces a transmembrane orientation of the poreforming peptide lantibiotic nisin. Biochemistry 41, 12171-12178

20. Breukink, E., van Heusden, H. E., Vollmerhaus, P. J., Swiezewska, E., Brunner, L., Walker, S., Heck, A. J. R., and de Kruijff, B. (2003) Lipid II is an intrinsic component of the pore induced by nisin in bacterial membranes. J. Biol. Chem. 278, 19898-19903

21. Kuipers, O. P., Rollema, H. S., Yap, W. M. G. J., Boot, H. J., Siezen, R. J., and de Vos, W. M. (1992) Engineeing dehydrated aminoacid residues in the antimicrobial peptide nisin. J. Biol. Chem. 267, 24340-24346

22. Dottavio-Martin, D., and Ravel, J. M. (1978) Radiolabeling of proteins by reductive alkylation with (C-14) formaldehyde and sodium cyanoborohydride. Anal. Biochem. 87, 562-565

23. Swiezewska, E., Sasak, W., Mankowski, T., Jankowski, W., Vogtman, T., Krajewska, I., Hertel, J., Skoczylas, E., and Chojnacki, T. (1994) The search for plant polyprenols. Acta Biochim. Pol. 41, 221-260

24. Haeberlen, U., and Waugh, J. S. (1969) Spin-lattice relaxation in periodically perturbed systems. Phys. Rev. 185, 420-429

25. Hartmann, S. R., and Hahn, E. L. (1962) Nuclear double resonance in rotating frame. Phys. Rev. 128, 2042-2053

26. Bennet, A. E., Reinstra, C. M., Auger, M., Lakshami, K. V., and Griffin, R. G. (1995) Heteronuclear decoupling in rotating solids. J. Chem. Phys. 103, 6951-6958

27. Guillion, T., and Schaefer, J. (1989) Rotational-echo double resonance. J. Magn. Res. 81, 196-200

28. Bonev, B. B., Watts, A., Bokvist, M., and Grobner, G. (2001) Electrostatic peptide-lipid interactions of amyloid $\beta$-peptide and pentalysine with membrane surfaces monitored by ${ }^{31} \mathrm{P}$ MAS NMR. Phys. Chem. Chem. Phys. 3, 2904-2910

29. Hsu, S.-T., Breukink, E., de Kruijff, B., Kaptein, R., Bonvin, A. M. J. J., and van Nuland, N. A. J. (2002) Mapping the targeted membrane pore formation mechanism by solution NMR: The nisin $\mathrm{Z}$ and Lipid II interaction in SDS micelles. Biochemistry 41, $7670-7676$

30. Kuipers, O. P., Rollema, H. S., de Vos. W. M., and Siezen, R. J. (1993) Biosynthesis and secretion of a precursor of nisin-Z by Lactococcus lactis, directed by the leader peptide of the homologous lantibiotic subtilin from Bacillus-subtilis. FEBS Lett. 330, 23-27

Received for publication May 14, 2004. Accepted for publication August 5, 2004. 